\title{
Growth performance and immune response of two commercial broiler strains fed diets containing Lactobacillus cultures and oxytetracycline under heat stress conditions
}

\begin{abstract}
1. Hubbard $\times$ Hubbard $(\mathrm{HH})$ and Shaver $\times$ Shaver $(\mathrm{SS})$ chicks given a dietary supplement of either $50 \mathrm{mg} / \mathrm{kg}$ oxytetracycline (OTC) or $1 \mathrm{~g} / \mathrm{kg}$ Lactobacillus culture (LC) were exposed to $36 \pm 1{ }^{\circ} \mathrm{C}$ for $3 \mathrm{~h}$ daily from day (d) 21 to 42 . 2. Prior to heat treatment, body weight (d 21) and weight gain ( 1 to $\mathrm{d} 21$ ) of OTC and LC birds were greater than those fed the control diet. Chicks given LC had the best food efficiency followed by OTC and control birds during $\mathrm{d} 1$ to $\mathrm{d} 21$. Body weight ( 1 and d 21) and weight gain (d 1 to $\mathrm{d} 21$ ) were greater for $\mathrm{HH}$ than SS chicks. 3. After 3 weeks of heat exposure, birds receiving the LC diet had greater body weight and weight gain, higher food intake and lower food efficiency than OTC and control chicks. 4. Antibody production against Newcastle disease vaccine on $\mathrm{d} 21$ was not affected by strain or diet. On d 42, while diet had negligible effect on this variable among the SS broilers, HH birds fed LC had higher antibody production than those on the control diet. 5. Neither strain nor diet had a significant effect on mortality.
\end{abstract}

Keyword: Oxytetracycline; Lactobacillus culture; Chickens; Newcastle disease virus; Antibacterial agents; Antibodies 